\title{
REDEFINING THE NEED OF CT SCAN IN CHRONIC RHINOSINUSITIS- A RATIONAL APPROACH
}

\author{
Soumik Basuㄹ, Pranay Kumar Agarwal ${ }^{2}$
}

${ }^{1}$ Senior Resident, Department of ENT, IPGMER, Kolkata, West Bengal, India.

${ }^{2}$ Senior Resident, Department of ENT, IPGMER, Kolkata, West Bengal, India.

\begin{abstract}
BACKGROUND
ABSTRACT

Chronic rhinosinusitis is a global health problem affecting a large population of people, thus causing significant morbidity. Its diagnosis has remained a dilemma and clinicians need to resort to expensive investigations such as CT scan for a confirmed diagnosis, thus increasing healthcare costs. It is imperative to reduce the cost of healthcare in developing nations, as a large number of patients cannot pay for expensive investigations. Nasal endoscopy appears to be a cheaper alternative to CT scans for the diagnosis of chronic rhinosinusitis and its use has gained prominence in recent times. The aim of this study is to see if there is any correlation between the findings of nasal endoscopy and CT scan findings of patients suspected to have chronic rhinosinusitis diagnosed by symptomatology according to EPOS 2012 criterion and formulate an efficient management plan based on the results.
\end{abstract}

\section{METHODS}

Patients presenting to the ENT OPD and meeting clinical criteria of chronic rhinosinusitis under EPOS 2012 were selected and subjected to nasal endoscopy and CT scan of the nose \& paranasal sinuses. Endoscopic findings were then graded according to Lund-Kennedy score, while the CT scan findings were graded according to Lund-Mackay score. These were then statistically analysed.

\section{RESULTS}

A total of 55 patients were selected. The mean Lund-Kennedy score was 5.36 with a standard deviation of 3.628. The mean LundMackay score was 9.91 with a standard deviation of 6.375. There was a positive correlation between nasal endoscopic and CT scan findings, which was statistically significant $(\mathrm{p}<0.005)$. The sensitivity and specificity of nasal endoscopy to diagnose chronic rhinosinusitis was $93.61 \%$ and $62.5 \%$ respectively.

\section{CONCLUSIONS}

Nasal endoscopy is highly sensitive in diagnosing chronic rhinosinusitis and should be the investigation of choice in patients suspected to have chronic rhinosinusitis. CT scan may be reserved for patients who are endoscopically negative or who are planning to undergo operative management. This can cause a significant reduction in the costs associated with the management of chronic rhinosinusitis as well as decrease the radiation exposure associated with CT scans.

\section{KEY WORDS}

Chronic Rhinosinusitis, Nasal Endoscopy, CT Scan, Lund Kennedy Score, Lund Mackay Score

HOW TO CITE THIS ARTICLE: Basu S, Agarwal PK. Redefining the need of CT scan in chronic rhinosinusitis- a rational approach. J. Evolution Med. Dent. Sci. 2019;8(30):2412-2416, DOI: 10.14260/jemds/2019/528

\section{BACKGROUND}

Chronic rhinosinusitis is a very common problem worldwide causing significant morbidity and healthcare expenditure.1,2 It is characterized by inflammation of the mucosa of the nose and paranasal sinuses lasting more than 12 weeks. Although, previously the term "sinusitis" was used, as "rhinitis" and "sinusitis" coexist, the term "rhinosinusitis" is now preferred.

In 1997, the Rhinosinusitis Task Force Committee of The American Academy of Otolaryngology-Head and Neck Surgery came out with definitions of rhinosinusitis which was based on subjective criterion. ${ }^{3}$

'Financial or Other Competing Interest': None.

Submission 13-05-2019, Peer Review 10-07-2019,

Acceptance 17-07-2019, Published 29-07-2019.

Corresponding Author:

Dr. Pranay Kumar Agarwal,

\#34, Ballygunge Circular Road,

Devadwar Apartment, Flat 2G,

Kolkata-700019,

West Bengal, India.

E-mail: pranaykagarwal@gmail.com

DOI: $10.14260 /$ jemds $/ 2019 / 528$
These subjective diagnostic criteria required at least two major factors or one major and two minor factors for diagnosing chronic rhinosinusitis. The major factors included facial pain or pressure, facial congestion or fullness, nasal obstruction or blockage, nasal discharge or purulence or discoloured post nasal discharge, hyposmia or anosmia, and purulence in nasal cavity on examination. The minor factors included headache, fever, halitosis, fatigue, dental pain, cough and ear pain or pressure or fullness.

However, as the classification of rhinosinusitis was based only on subjective symptoms, it was felt that the specificity and predictive value of Task Force criteria was not adequate to serve as diagnostic standard and additional validating data was needed ${ }^{4,5}$. Sinus Allergy Health Partnership (SHAP) task force revised the criterion in 2004 and stressed the need for objective evaluation for confirming the diagnosis of rhinosinusitis 6 .

This need for objective evaluation led to the inclusion of Nasal endoscopy and Computed Tomography Scans to support the diagnosis of chronic rhinosinusitis by the European Position Paper on Rhinosinusitis and Nasal Polyps. 


\begin{tabular}{|c|c|c|}
\hline CT Scan Finding & No. of Patients & Percentage (\%) \\
\hline Blockage of Ostiomeatal Complex & $36 / 55$ & $65.45 \%$ \\
\hline Opacity of Maxillary Sinus & $45 / 55$ & $81.81 \%$ \\
\hline Opacity of Anterior Ethmoids & $38 / 55$ & $58.18 \%$ \\
\hline Opacity of Posterior Ethmoids & $22 / 55$ & $40 \%$ \\
\hline Opacity of Frontal Sinus & $20 / 55$ & $36.36 \%$ \\
\hline Opacity of Sphenoid Sinus & $11 / 55$ & $20 \%$ \\
\hline \multicolumn{2}{|c|}{ Table 2. CT Scan Findings } \\
\hline
\end{tabular}
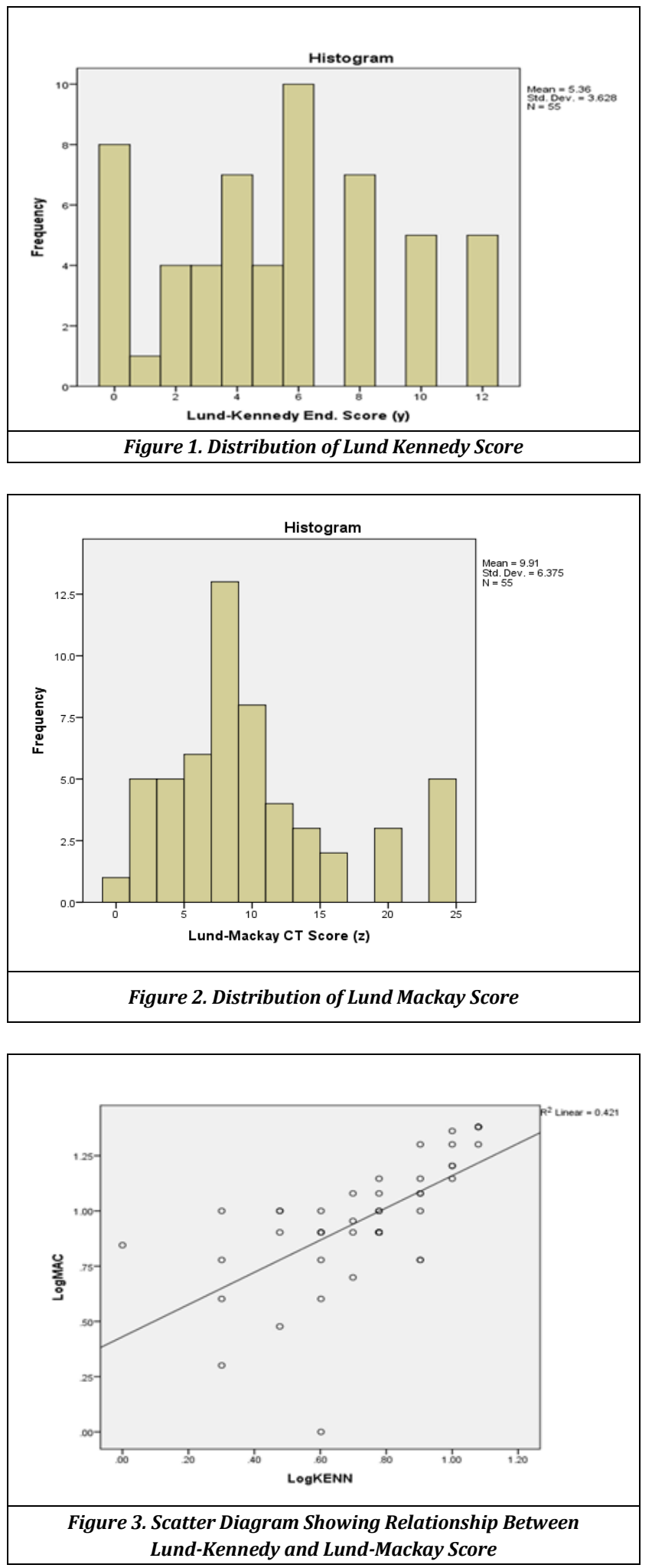

\section{RESULTS}

A total of 55 patients were enrolled in this study, out of which 25 were male $(45.45 \%)$ and 30 were female $(54.54 \%)$ with a Male: Female ratio is $1: 1.2$. On nasal endoscopy, 47 patients were Endo ( + ) and 8 were Endo (-). The mean Lund-Kennedy score was 5.36 with a standard deviation of 3.628 The presentation of disease is shown in table 1. The CT Scans were scored using the Lund Mackay staging system. A Lund Mackay score of three or less was considered normal. Opacity of the maxillary sinus was the most common finding on CT scan $(81.8 \%)$, followed by anterior ethmoids. The mean Lund Mackay score was 9.91 with a standard deviation of 6.375 . The CT scan findings are shown in Table 2. Among the 55 patients who were diagnosed with chronic rhinosinusitis based on their symptoms, nasal endoscopy was abnormal in 47 patients $(85.45 \%)$. Of these 47 patients, CT scan was abnormal in 44 patients and normal in 3. Eight patients had normal endoscopy; of these, 3 patients had abnormal CT scan and 5 patients had normal CT scan. The distribution pattern of the variables was determined for the sample size of 55 $(n=55)$. The Kolmogorov- Smirnov test was used. Parametric tests were done for both the variables (Lund Kennedy score and Lund Mackay score) and the Pearson coefficient(r) was determined. There was a statistically significant positive correlation between them $(\mathrm{r}=0.649, \mathrm{p}=0.000)$. Taking CT scan as gold standard, the sensitivity of endoscopy to diagnose chronic rhinosinusitis was $93.61 \%$ with the specificity being $62.5 \%$.

\section{DISCUSSION}

Chronic rhinosinusitis is a very common ENT disease presenting in the Outpatient department. Even though a detailed history taking is very important for the diagnosis of chronic rhinosinusitis, its accurate diagnosis cannot be made just based on symptomatology. Most of the guidelines now routinely recommend the use of Nasal endoscopy and/ or CT Scans to aid the diagnosis. ${ }^{1}$

For our study, we enrolled patients who were suspected to have chronic rhinosinusitis based on their history. We found the male: female ratio as 1:1.2. We subjected the patients to both nasal endoscopy and CT scan of the nose \& paranasal sinuses. During Nasal endoscopy, we found oedema of the nasal mucosa to be the most common finding and affected $76.36 \%$ of individuals. This was followed by nasal discharge. This is in stark contrast to the study conducted by Shahizon AM et al. ${ }^{9}$ They had found polyps to be the most common presentation followed by mucosal disease. This difference in findings may be due to use of excessive nasal packing and decongestion prior to nasal endoscopy that may significantly reduce mucosal oedema, which is frequently a precursor to polyp formation. Vining et $\mathrm{al}^{10}$ studied the preoperative importance of endoscopic assessment of nasal cavity of patients with chronic rhinosinusitis, and concluded that nasal endoscopy helped to determine the type of soft tissue obstructing the middle meatus. It also demonstrated other findings such as septal deviations, adenoid hypertrophy and turbinate enlargement.

On CT scan, the most common sinus to be involved was maxillary sinus followed by anterior ethmoids. It was interesting to note that all cases with Ostiomeatal complex (OMC) blockage had opacity in maxillary and anterior ethmoids. This is probably since both these sinuses drain through the narrow OMC, any oedema in this area leads to disruption of mucociliary clearance and stasis of nasal secretions leading to sinusitis. 


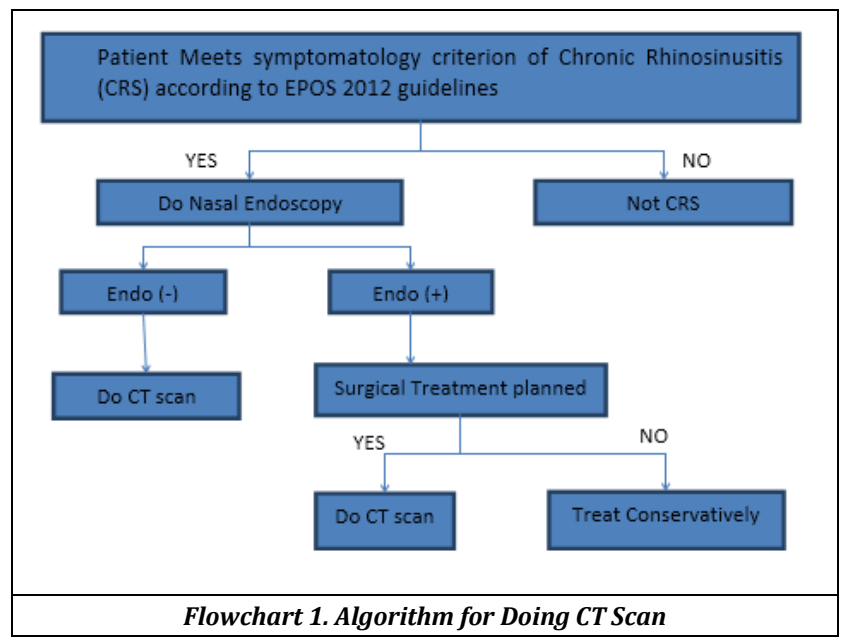

Bhattacharya et $\mathrm{al}^{11}$ and Bradley DT et $\mathrm{al}^{12}$ tried to compare the symptom score of patients with chronic rhinosinusitis and the CT scan score, but found no significant relation between them.

We compared the findings of nasal endoscopy and CT scan. Nasal endoscopy was better at detecting nasal polyps and discharge. These findings could not be seen on the CT scan as CT scan just showed an opacity which could be due to discharge, polyps or mucosal oedema. However, CT scan was better at evaluating the sinuses. Posterior ethmoids and frontal sinus could only be evaluated using CT Scans. CT scans were also better at delineating anatomical details required for surgical procedures. Even though CT scan is considered a gold standard for diagnosing chronic rhinosinusitis, it has some inherent problems. It is not widely available in a resource poor country like India. Where available, the quality of scans is debatable. Routine use of CT scans for diagnosing chronic rhinosinusitis is not a cost-effective solution in a developing nation. Exposure to harmful radiation is of concern and so the use of CT scans should be minimized as much as possible. To address the above issues, we did a statistical analysis which showed a positive correlation between the nasal endoscopy and CT scan findings ( $r=0.649$, $\mathrm{p}=0.000$ ). In the study by Pokharel et al ${ }^{13}$ also there was a statistically significant correlation $(\mathrm{r}=0.556)$ between endoscopic and CT scan scores.

In our study nasal endoscopy showed a high sensitivity of 93.6\% to diagnose chronic rhinosinusitis but it had a low specificity of only $62.5 \%$. Kolethekkat et al, had found the sensitivity and specificity to be $91 \%$ and $44 \%$ respectively ${ }^{14}$, whereas Deosthale NV et al ${ }^{15}$ found them to be $94 \%$ and $75 \%$ respectively. As the sensitivity of nasal endoscopy is very high, it is very less likely to miss the diagnosis of chronic rhinosinusitis. This shows that nasal endoscopy can be used as a first line investigation for patients suspected to have chronic rhinosinusitis.

As nasal endoscopy is cheap, without associated radiation risks and also very highly sensitive for diagnosis of chronic rhinosinusitis, we propose that CT scan should be reserved for patients suspected to have chronic rhinosinusitis based on symptomatology but are found to be Endo (-). However, if surgical treatment is being planned for the patient, then the patient should undergo a non-contrast CT scan so as to delineate the anatomy better, which will lead to a safer surgery. A working flowchart of the same is outlined below-

\section{Ethical Approval}

Ethical approval was taken from the Institutional Ethics Committee. All procedures and tests performed on human participants were in accordance to the ethical standards of the institutional ethics committee.

\section{CONCLUSIONS}

Both, nasal endoscopy and CT scan of paranasal sinuses have a role to play in the management of chronic rhinosinusitis and are positively correlated. Although CT scan gives a more detailed anatomical layout of the nose and paranasal sinuses, endoscopy is an excellent alternative for diagnosis and helps to decrease the cost of treatment in resource poor countries. Also, it decreases the radiation exposure a patient receives. Endoscopy can be the first choice of investigation in patients suspected of having chronic rhinosinusitis, with CT scan reserved for those patients who have normal endoscopic findings or are undergoing sinus surgery. Additional studies with a larger sample size would be required to further validate the proposed management and the cost benefit analysis of the same.

\section{REFERENCES}

[1] Fokkens W, Lund V, Mullol J, et al. European position paper on rhinosinusitis and nasal polyps 2007. Rhinol Suppl 2007;20:1-136.

[2] Fokkens WJ, Lund VJ, Mullol J, et al. European position paper on rhinosinusitis and nasal polyps 2012. Rhinol Suppl 2012;(23):1-298.

[3] Lanza DC, Kennedy DW. Adult rhinosinusitis defined. Otolaryngol Head Neck Surg 1997;117(3 Pt 2):S1-S7.

[4] Stankiewicz JA, Chow JM. A diagnostic dilemma for chronic rhinosinusitis: definition accuracy and validity. Am J Rhinol 2002;16(4):199-202.

[5] Hwang PH, Irwin SB, Griest SE, et al. Radiologic correlates of symptom-based diagnostic criteria for chronic rhinosinusitis. Otolaryngol--Head Neck Surg 2003;128(4):489-96.

[6] Meltzer EO, Hamilos DL, Hadley JA, et al. Rhinosinusitis: establishing definitions for clinical research and patient care. J Allergy Clin Immunol 2004;114(6 Suppl):155-212.

[7] Lund VJ, Kennedy DW. Quantification for staging sinusitis. The Staging and Therapy Group. Ann Otol Rhinol Laryngol Suppl 1995;167:17-21.

[8] Lund VJ, Mackay IS. Staging in rhinosinusitus. Rhinology 1993;31(4):183-4.

[9] Shahizon AMM, Suraya A, Rozmnan Z, et al. Correlation of computed tomography and nasal endoscopic findings in chronic rhinosinusitis. Med J Malaysia 2008;63(3):211-5.

[10] Vining EM, Yanagisawa K, Yanagisawa E. The importance of preoperative nasal endoscopy in patients with sinonasal disease. The Laryngoscope 1993;103(5):512-9. 
[11] Bhattacharyya T, Piccirillo J, Wippold FJ 2nd. Relationship between patient-based descriptions of sinusitis and paranasal sinus computed tomographic findings. Arch Otolaryngol Head Neck Surg 1997;123(11):1189-92.

[12] Bradley DT, Kountakis SE. Correlation between computed tomography scores and symptomatic improvement after endoscopic sinus surgery. The Laryngoscope 2005;115(3):466-9.

[13] Pokharel M, Karki S, Shrestha BL, et al. Correlations between symptoms, nasal endoscopy computed tomography and surgical findings in patients with chronic rhinosinusitis. Kathmandu Univ Med J (KUMJ) 2013;11(43):201-5.
[14] Kolethekkat AA, Paul RR, Kurien M, et al. Diagnosis of adult chronic rhinosinusitis: can nasal endoscopy predict intrasinus disease? Oman Med J 2013;28(6):427-31.

[15] Deosthale NV, Khadakkar SP, Harkare VV, et al. Diagnostic accuracy of nasal endoscopy as compared to computed tomography in chronic rhinosinusitis. Indian J Otolaryngol Head Neck Surg 2017;69(4):4949. 